Onkologe 2018 $\cdot 24: 368-377$ https://doi.org/10.1007/s00761-018-0344-9 Online publiziert: 13. Februar 2018 (c) Der/die Autor(en) 2018. Dieser Artikel ist eine Open-Access-Publikation.

CrossMark

\author{
Sebastian Kuhn ${ }^{1} \cdot$ Dennis Kadioglu ${ }^{2,3} \cdot$ Kim Deutsch $^{4} \cdot$ Susanne Michl ${ }^{5}$ \\ 'Zentrum für Orthopädie und Unfallchirurgie, Universitätsmedizin der Johannes Gutenberg-Universität, \\ Mainz, Deutschland \\ ${ }^{2}$ Medical Informatics Group (MIG), Universitätsklinikum Frankfurt, Frankfurt am Main, Deutschland \\ ${ }^{3}$ Medizinische Informatik, IMBEl, Universitätsmedizin der Johannes Gutenberg-Universität Mainz, Mainz, \\ Deutschland \\ ${ }^{4}$ Zentrum für Orthopädie und Unfallchirurgie, Universitätsmedizin der Johannes Gutenberg-Universität, \\ Mainz, Deutschland \\ ${ }^{5}$ Institut für Geschichte der Medizin und Ethik in der Medizin, Charité - Universitätsmedizin Berlin, Berlin, \\ Deutschland
}

\title{
Data Literacy in der Medizin
}

\section{Welche Kompetenzen braucht ein Arzt?}

\section{Die zunehmende Digitalisierung der Arbeits- und Lebenswelt hat auch die Medizin erreicht. Bereits heute verändert der Einsatz vielfältiger digitaler Techniken in medizinischer Versorgung und Forschung den Beruf des Arztes.}

\section{Von der Medizin zur Datenwissenschaft}

In der Onkologie erhofft man sich durch gezielten Einsatz der technischen Möglichkeiten hinsichtlich Sequenzierung und bioinformatischer Analyse sowie der anschließenden fächerübergreifenden Ergebnisauswertung neue, besser an den individuellen Patienten angepasste Behandlungskonzepte [1]. In einer Initiative des Bundesministeriums für Bildung und Forschung (BMBF) wird das Ziel einer individualisierten Medizin fächerübergreifend als grundlegende Strategie verfolgt [2]. Noch einen Schritt weiter geht die Medizininformatikinitiative des BMBF, welche unter der sehr allgemeinen Zielsetzung „Digitalisierung in der Medizin" anstrebt, das deutsche Gesundheitswesen digital zu vernetzen und so dafür zu sorgen, dass die Vielzahl an bereits heute gesammelten Daten einfacher und sinnvoller genutzt werden kann [3].

All diesen Vorhaben gemein ist die Tatsache, dass den Ärzten zukünftig eine um ein Vielfaches größere Datenmenge zur Verfügung stehen wird, die bei der Entscheidungsfindung berücksich- tigt werden muss. Längst unterstützen Computersysteme die Ärzte dabei, für ihre Patienten Therapieentscheidungen zu treffen, wie z. B. das Cognitive-Computing-System IBM Watson for Oncology [4]. Die endgültige Entscheidung liegt aber nach wie vor beim Menschen, sprich dem Arzt, der in Folge dessen die notwendigen Kompetenzen besitzen muss, um einerseits die Daten und andererseits die darauf beruhenden Entscheidungsfindungsprozesse verstehen und richtig einschätzen zu können.

\section{》) Mithilfe von Smartphone-} Apps gelangen z. B. Anamnesesysteme in Form eines Chatbots zum Patienten

Mit Lösungen wie der Smartphone-App Ada-Health finden aufkünstlicher Intelligenz (KI) basierende Anamnesesysteme in Form eines Chatbots den Weg in Patientenhände [5]. Der Patient von morgen, ausgestattet mit solchen Apps und durch Quantified-Self im Bild über seine eigenen Lebensparameter, verlangt vom Arzt der Zukunft, bei Diagnostik und Therapie auch solche Daten aus externen Quellen richtig einschätzen und entsprechend berücksichtigen zu können.

\section{Implikationen für die medizinische Ausbildung}

Vor dem Hintergrund aktueller Diskussionen über den Arztberuf mit einer Neudefinition des professionellen Rollenverständnisses, der Kompetenzorientierung sowie des interdisziplinären und multiprofessionellen Arbeitens ist die Integration von Data Literacy in das Medizinstudium von großer Bedeutung [6]. Eine solche didaktische Umsetzung erscheint umso dringlicher, als empirische Studien gezeigt haben, dass die erste Generation der Digital Natives zwar auf der Konsumentenebene mit digitalen Medien bestens vertraut ist, berufsspezifische digitale Handlungskompetenzen jedoch kaum ausgebildet sind [7]. Es ist essenziell, dem veränderten Qualifikationsprofil der angehenden Ärzte Rechnung zu tragen und entsprechende Lehrinhalte zentral im Curriculum zu verankern $[8,9]$. Aber worin die Kompetenzen eines Arztes im digitalen Zeitalter bestehen und wie diese gelehrt werden können, ist aktuell weitestgehend nicht geklärt. Der Beitrag möchte deswegen die Kompetenzen definieren, die im Begriff der Data Literacy im medizinischen Kontext gebündelt werden, und ein konkretes Lehrkonzept „Medizin im digitalen Zeitalter" vorstellen, das an der Universitätsmedizin Mainz zum Sommersemester 2017 implementiert wurde. 
Hier steht eine Anzeige.

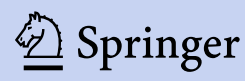


Onkologe 2018 $24: 368-377$ https://doi.org/10.1007/s00761-018-0344-9

(c) Der/die Autor(en) 2018. Dieser Artikel ist eine Open-Access-Publikation.

\section{S. Kuhn · D. Kadioglu $\cdot$ K. Deutsch $\cdot$ S. Michl}

\section{Data Literacy in der Medizin. Welche Kompetenzen braucht ein Arzt?}

\section{Zusammenfassung}

Hintergrund. Die digitale Transformation des Gesundheitssystems verändert den Beruf des Arztes. Data Literacy wird hierbei als eine der führenden Zukunftskompetenzen erachtet, findet jedoch derzeit weder in den implementierten Curricula des Medizinstudiums noch in den aktuell laufenden Reformprozessen (Masterplan Medizinstudium 2020 und Nationaler Kompetenzbasierter Lernzielkatalog) Beachtung.

Ziel. Der Beitrag möchte zum einen die Aspekte beleuchten, die im Begriff der Data Literacy im medizinischen Kontext gebündelt werden. Zum andern wird ein Lehrkonzept vorgestellt, das Data Literacy im Zeichen der digitalen Transformation erstmals im Medizinstudium abbildet.
Material und Methoden. Das BlendedLearning-Curriculum "Medizin im digitalen Zeitalter" adressiert in 5 Modulen den diversen Transformationsprozess der Medizin von digitaler Kommunikation über Smart Devices und medizinische Apps, Telemedizin, virtuelle/augmentierte und robotische Chirurgie bis hin zu individualisierter Medizin und Big Data. Diese Arbeit stellt Konzept und Erfahrungen der erstmaligen Implementierung des 5. Moduls dar, welches transdisziplinär und integrativ den Aspekt Data Literacy erläutert.

Ergebnisse. Die Evaluation des Kurskonzepts erfolgte sowohl qualitativ als auch quantitativ und demonstriert einen Kompetenzgewinn in den Bereichen Wissen und Fertigkeiten sowie eine differenziertere Haltung nach Kursabschluss.

Schlussfolgerungen. Die curriculare Integration von Data Literacy ist eine transdisziplinäre und longitudinale Aufgabe. Bei der Entwicklung dieser Curricula sollten die hohe Geschwindigkeit des Veränderungsprozesses der digitalen Transformation beachtet und die curriculare Anpassung im Sinne eines Agility by Design bereits bei der Konzeption adressiert werden.

\section{Schlüsselwörter}

Digitale Transformation · Blended-LearningCurriculum · Medizinische Ausbildung · Big Data · Digitale Ethik

\section{Data literacy in medicine. What competences does a physician need?}

\section{Abstract}

Background. The digital transformation of healthcare is changing the profession of physicians. "Data Literacy" is considered to be one of the most relevant future competences. Currently "Data Literacy" is not addressed in German medical school curricula and also the current ongoing reform processes (Master Plan for Medical Studies 2020 and National Competency-Based Learning Objective Catalog) do not address this field. Objectives. First, the article aims to address the concept of "Data Literacy" in the medical and educational context. Secondly, a teaching concept will be presented that depicts "Data Literacy" in medicine for the first time in a German medical school.

\begin{abstract}
Materials and methods. The blended learning curriculum "Medicine in the Digital Age" addresses in five modules the diverse transformation process of medicine ranging from digital communication, smart devices and medical apps, telemedicine, virtual/augmented and robotic surgery to individualized medicine and big data. This publication presents the teaching concept, the initial implementation and the evaluation of the fifth module, which focuses on "Data Literacy" in a transdisciplinary and integrative way.

Results. The evaluation of the new course concept was conducted qualitatively and quantitatively. The results demonstrate a gain
\end{abstract}

in competency in the areas of "knowledge" and "skills" as well as a more differentiated "attitude".

Conclusions. The curricular integration of "Data Literacy" is a transdisciplinary and longitudinal task. However, the high speed of the transformation process needs to be considered for the development of these curricula. An adaptation option in the sense of "Agility by Design" is hereby a purposeful approach.

\section{Keywords}

Digital transformation · Blended learning curriculum - Medical education - Big data . Digital ethics

\section{Digital Literacy und Data Literacy}

Mitte der 1990er Jahre prägte der Publizist Gilster den Begriff Digital Litera$c y$, um diejenigen Kompetenzen zu bestimmen, die in einer digitalisierten Arbeits- und Lebenswelt erworben werden müssen [10, 11]. Gilster präsentierte in seinem gleichnamigen Buch keine Liste an spezifischen technischen Fertigkeiten wie einige Autoren vor ihm, sondern sprach von einer weiter gefassten Kompetenz, „Ideen zu beherrschen und nicht nur Tastenanschläge“. Literacy ist demnach mehr als nur das Kodieren und Dekodieren von Zeichen, sondern eine kulturelle Praktik wie sie auch für papierbasierte Medien erlernt werden muss. Sie erfasst im weiteren Sinne alle Erfahrungen und Grundfertigkeiten rund um Erzähl-, Sprach- und Schriftkultur, wie Textverständnis und Sinnverstehen, sprachliche Abstraktionsfähigkeit, schriftlichen Ausdruck und die Vertrautheit mit Schriftsprache. Auch beim Einsatz von digitalen Medien, Daten und Informationen muss diese kulturelle Praktik erlernt werden.
Dies wird umso wichtiger, je mehr Daten die Grundlage von Entscheidungen und Handlungsoptionen sind, weswegen im Folgenden der Begriff Data Literacy vor dem deutlich breiter gefassten Begriff Digital Literacy bevorzugt wird.

In der aktuellen Medizinerausbildung wird der Umgang mit Daten zumeist im Kontext der statistischen Grundausbildung gelehrt, um die zukünftigen Ärzte in die Lage zu versetzen, Forschungsergebnisse medizinischer Studien und Veröffentlichungen richtig einschätzen zu können. Ein Lehrkonzept, welches Data Li- 
Hier steht eine Anzeige.

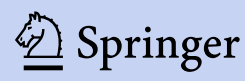


teracy als Kernkompetenz ernst nimmt, muss sich demgegenüber jedoch zusätzlich auf die Vermittlung von digitalen Fertigkeiten bezüglich eines kritischen, planvollen und kontextspezifischen Umgangs mit Daten fokussieren. Daten müssen somit nicht nur ausgewertet und Störvariablen ausgeschaltet werden. Sie müssen verwaltet, kuratiert, analysiert, visualisiert, interpretiert und kontextualisiert werden, um nicht nur als statistisch valide, sondern auch als ethisch gute und rechtlich sichere Entscheidungs- und Handlungsgrundlage $\mathrm{zu}$ dienen. Nicht zuletzt bedeutet ein kritischer Umgang auch, die Grenzen der Aussagekraft von datenbasierten Entscheidungen zu kennen. Welche Erkenntnisse bleiben uns verschlossen, weil die Antwort auf alle offenen Fragen nicht immer durch quantifizierbare Parameter abgebildet werden kann? Neben spezialisierten Fachkräften wie den Data Scientists brauchen wir in allen Sektoren Fachkräfte, die über Data-Literacy-Kompetenzen verfügen. Die Entwicklung dahingehender curricularer Konzepte und deren Implementierung steht, mit Ausnahme weniger Beispiele, allerdings noch ganz am Anfang [12-14]. Ein so definierter erweiterter Kompetenzerwerb entsteht zudem nicht einfach als „Nebenprodukt einer fachlichen Wissensvermittlung“, sondern muss in einem Kurskonzept abgebildet werden, das gezielt und systematisch diesem veränderten ärztlichen Qualifikationsprofil Rechnung trägt.

Das Hochschulforum Digitalisierung hat $\mathrm{zu}$ diesen Aspekten im Sommer 2017 eine Arbeitsgruppe Curriculum 4.0 - Curriculumentwicklung im 21. Jahrhundert gegründet [15]. Eine aktuell laufende Studie dieser Arbeitsgruppe soll im Frühjahr 2018 erste Antworten liefern, inwiefern Data Literacy zukünftig immersiv in alle Disziplinen und Curricula integriert werden kann und welche Modelle der Integration sich im internationalen Umfeld bereits bewährt haben [16]. Bei dieser Neuentwicklung muss jedoch die hohe Geschwindigkeit des Veränderungsprozesses im Sinne eines Agility by Design adressiert werden.

\section{Unterrichtskonzept}

Vom 29.05. bis 02.06.2017 fand die erstmalige Implementierung von Medizin im digitalen Zeitalter an der Universitätsmedizin Mainz statt. Das einwöchige Wahlpflichtfach setzte sich aus 5 Lernmodulen zusammen, die jeweils aus einer E-Learning-Einheit und einer 3-stündigen Präsenzunterrichtseinheit bestanden:

- Modul 1 - Digitale Arzt-Patienten-Kommunikation und soziale Netzwerke,

- Modul 2 - Smart Devices und medizinische Apps,

- Modul 3 - Telenotarzt, Teleradiologie, Telemedizin,

- Modul 4 - Virtual Reality, Augmented Reality und computerassistierte Chirurgie,

- Modul 5 - Individualisierte Medizin und Big Data.

Das Blended-Learning-Curriculum Medizin im digitalen Zeitalter zielt bewusst auf eine Mischform zwischen digitalen Lehr- und Lernformen sowie Präsenzunterricht $a b$. Vor Unterrichtsbeginn erarbeiten die Studierenden anhand eines eBook Grundlagen der digitalen Medizin. Da zum Erreichen einer Ausbildungsexzellenz sowohl die kognitive und affektive Ausbildungsebene als auch die Ebene der praktischen Fertigkeiten einbezogen werden müssen, berücksichtigt der Präsenzunterricht ein Lernen durch Erleben und ein kollaboratives Arbeiten [17]. Eine intensive Zusammenarbeit zwischen Studierenden und Dozierenden bildet das Grundverständnis des Kurses. Der Präsenzunterricht wird in Kleingruppen mit Unterstützung verschiedener medizinischer Fachdisziplinen (Anästhesie, Chirurgie, medizinische Informatik Medizinethik, Psychologie, Pädiatrie, Psychosomatik, Radiologie, Unfallchirurgie und Orthopädie) durchgeführt. Des Weiteren wird das Dozententeam im Sinne eines transdisziplinären Ansatzes durch AppEntwickler und Patienten erweitert. Die Studierenden benennen am jeweiligen Kurstag ihre persönlichen Lernziele und überprüfen zum Modulende, ob diese erreicht wurden. Ein digitales Kommunikationstool $\left(\mathrm{SLACK}^{\circledR}\right)$ wurde über die gesamte Kursdauer als Plattform für Diskussion sowie Informationsaustausch verwendet und soll zum informellen Meinungs- und Erfahrungsaustausch anregen. Ziel ist es, die Studierenden $\mathrm{zu}$ aktivem und eigeninitiativem Lernen anzuleiten. Neben dem Erwerb von Wissen und spezifischen Fertigkeiten liegt ein weiterer Schwerpunkt auf der Motivation der Studierenden hin zu einer differenzierten Haltung und zur Selbstverortung der Studierenden in einer digitalen Medizinwelt.

Im Folgenden wird der Ablauf des abschließenden Modul 5 - individualisierte Medizin und Big Data beschrieben. Die gemeinsamen Diskussionen orientierten sich an den folgenden Fragekomplexen:

\section{Fragenkomplex 1: Welche Daten pro-} duzieren wir? Wenn Daten die Grundlage wichtiger (Therapie-)Entscheidungen und Handlungsoptionen sind, so muss gewährleistet sein, dass wir valide Daten produzieren. Welche Herausforderungen bei der Datenerhebung, -sicherung und -zusammenführung stellen sich uns aktuell und zukünftig?

$\mathrm{Zu}$ unterscheiden sind verschiedene Anlässe von Datensammlungen. In klinischen Studien werden Daten zielgerichtet auf eine Fragestellung hin gesammelt, entsprechend der Zielsetzung validiert und üblicherweise an einem spezifischen Ort gespeichert. Demgegenüber hinterlassen wir im Internet mit jedem Klick eine Unmenge an Metadaten, die weltweit dauerhaft gespeichert werden.

Fragenkomplex 2: Können Daten in der Medizin alles beantworten? Durch den Fokus auf große Datenmengen zentrieren sich Forschungsprioritäten auf Erkrankungen, welche durch große Zahlen erfasst und erforscht werden können. Was geschieht aber mit seltenen Erkrankungen oder Erkrankungen, welche nicht oder nicht so leicht nach klinischen, messbaren Parametern erfasst werden können (z.B. psychiatrische Erkrankungen)?

Fragenkomplex 3: Wie und von wem werden diese Daten benutzt? Datensammlungen sind niemals neutral. Sie werden zu einem bestimmten Zweck er- 


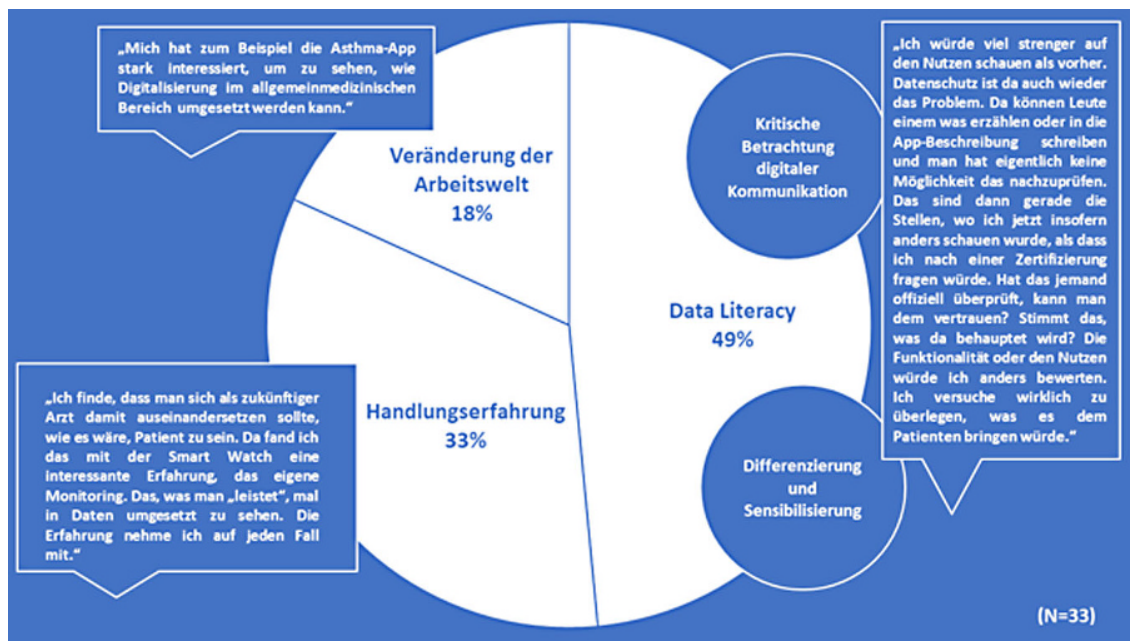

Abb. 1 A Ergebnisse der qualitativen Evaluation anhand semistrukturierter Interviews. Anteile der 3 Kategorien mit exemplarischen Zitaten der Studierenden

hoben bzw. für einen bestimmten Zweck genutzt („self-tracking“, klinische Studien, Metadaten zur Identifizierung und Klassifizierung im Internet). Wer hat den Nutzen, wer trägt die Nachteile?

Fragenkomplex 4: Bestimmen Daten nun wie wir leben? Wir stecken schon mitten in der digitalen Revolution, die unser Leben verändert. Dennoch müssen wir uns die Frage stellen, welche gesellschaftlichen Folgen daraus entstehen. Wie können wir etwa in einer Informations- und Wissensgesellschaft das Recht auf Nichtwissen sicherstellen? Möchte ich als junger Mensch mit 25 Jahren wissen, ob ich im Alter von 70 mit einer bestimmten Wahrscheinlichkeit an Alzheimer erkranken werde?

Wie kann sichergestellt werden, dass ein Patient, welcher sich nicht an der Datensammlung beteiligen will oder kann, das Recht zu diesem Entschluss behält und dabei keine negativen Auswirkungen auf die medizinische Behandlung fürchten muss?

\section{Fragenkomplex 5: Behalten wir unsere} Entscheidungshoheit und -verantwortung? Aufgrund der ständig zunehmenden Verfügbarkeit von Daten liegt der Vorteil von Entscheidungsunterstützung auf der Hand: Wir können unsere Entscheidungen viel fundierter treffen, wobei hier bewusst offengelassen wird, ob nun allein das Vorliegen der Informationen schon als Entscheidungs-

\section{Evaluation}

Die Evaluation des neuartigen Kurskonzepts erfolgte qualitativ in Form semistrukturierter Interviews und quantitativ anhand standardisierter Prä-/Post-Evaluationsfragebögen zu den Teilkompetenzen Wissen, Fertigkeiten und Haltung. Zur Erhebung der qualitativen Daten in Form von 45-minütigen semistrukturierten Interviews wurden alle 11 Teilnehmenden des Pilotkurses in insgesamt 5 Gruppen befragt. Die dabei angefertigten Audioaufnahmen wurden im Nachgang transkribiert und anhand des Verfahrens der Qualitativen Inhaltsanalyse nach Philipp Mayring ausgewertet. Die quantitative Evaluation zu Data Literacy wurde in Form einer Selbsteinstufung anhand einer siebenstufigen Likert-Skala von allen Teilnehmern vorgenommen.

unterstützung angesehen wird, oder erst durch eine dritte, die Daten verarbeitende Komponente - ein anderer Mensch oder ein Softwaresystem - von tatsächlicher Entscheidungsunterstützung gesprochen werden kann. Wieviel Information benötigen wir einerseits und wieviel können wir andererseits als Entscheidungsgrundlage überhaupt noch sinnvoll verarbeiten? Dürfen wir dann überhaupt noch Entscheidungen selbst treffen? Wer trägt die Verantwortung für eine Entscheidung?

Grundlage für die Diskussionen waren die vorhergehenden praxisnahen Lehreinheiten. Die Erfahrungen, die die Teilnehmenden dort gesammelt haben, mündeten in grundlegende Fragen, die nun systematisch zusammengefasst und in ihrer Komplexität nochmals aufgegriffen werden konnten. Das vorgestellte Lehrkonzept fußt auf dem Prinzip einer multiperspektivischen und transdisziplinären Annäherung an diese komplexen Fragen. Die Zusammensetzung der Dozierenden im 5. Modul aus Arzt, Medizininformatiker und Medizinethikerin rundeten das Gesamtbild für die Studierenden ab, dass zukünftige Herausforderungen einer digitalen Medizinwelt nur durch eine gesamtheitliche Betrachtung, unter Einbeziehung vielfältiger Expertisen, zu bewältigen sind.

\section{Qualitative Evaluation anhand semistrukturierter Interviews}

Im Gegensatz zur weit verbreiteten quantitativen Forschung eignet sich qualitative Forschung gut, um die Lebenswirklichkeit der Studierenden verstehen zu können. Durch die Analyse umfassender Interviews werden neben numerischen Häufigkeiten auch latente Sinnzusammenhänge aufgedeckt. Die Qualitative Inhaltsanalyse nach Philipp Mayring eignet sich für die Analyse und Auswertung der im Rahmen der Evaluation erhobenen und transkribierten Interviews, da sie nach einem sehr logischen, auf entscheidende Inhalte fokussierenden Muster verläuft. Die Inhalte werden hierbei schrittweise in spezifische Kategorien eingeteilt. Durch den Anteil des Textes, der einer Kategorie zugewiesen werden kann, können so im Nachhinein Sinnzusammenhänge, Wertungen und Relevanzen festgelegt werden [18].

Neben den reinen Inhalten liegt ein entscheidender Schwerpunkt auf dem Kommunikationszusammenhang, in dem das Gesagte stattfindet. So werden Sender, Gegenstand, soziokultureller Hintergrund, auffällige (wiederkehrende) Merkmale und die Zielgruppe nach Bedarf mit einbezogen $[18,19]$. Zunächst wird dafür eine inhaltliche Zusammenfassung angefertigt, in der das Material 


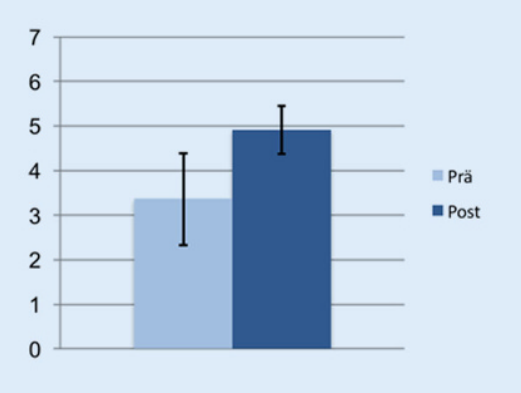

Abb. 2 \ Darstellung der Ergebnisse zur Frage "Wie schätzen Sie Ihr Wissen im Umgang mit individualisierter Medizin und Big Data ein?" im Prä-/Postvergleich ( 1 = sehr gering, $4=$ neutral, $7=$ sehr hoch)

soweit reduziert werden soll, dass die relevanten Textstellen hervortreten. Diese Reduzierung wird durch das Einsetzen von vorher erstellten Kategorien weitergeführt. Für unklare Abschnitte oder Textstellen gibt es die Möglichkeit einer explizierenden Inhaltsanalyse. Dieses Vorgehen zieht weiteres Material hinzu, um eben diese unklaren Abschnitte besser verstehen und in den richtigen Kontext setzen zu können. Unterschieden wird dabei in eine enge und weite Kontextanalyse. Die enge Kontextanalyse beschränkt sich auf die Informationen, die innerhalb des Interviews gefunden werden können. Die externe Kontextanalyse zieht externes, über die internen Interviewinformationen hinausreichendes Material hinzu, um weitere Rahmenbedingungen klären zu können [18].

Die Evaluation in Form der semistrukturierten Interviews zeigte eine hohe Akzeptanz des Kurskonzepts. Die Studierenden betonten dabei die empfundene Wertschätzung und Motivation durch die intensive und kreative Zusammenarbeit untereinander und mit den Dozierenden. Besonders die Möglichkeit der praktischen Interaktion und Verdeutlichung von medizinischen Versorgungskonzepten wurden dabei positiv bewertet. In allen erhobenen Interviews kam es zu einer Gesamtzahl von 33 Nennungen, die im Auswertungsverfahren der Kategorie Digital Literacy zugeordnet werden konnten. Diese Kategorie lässt sich des Weiteren in 3 Unterkategorien näher beschreiben. Mit 16 Nennungen bildet $D a$ ta Literacy die bedeutendste Unterkate-

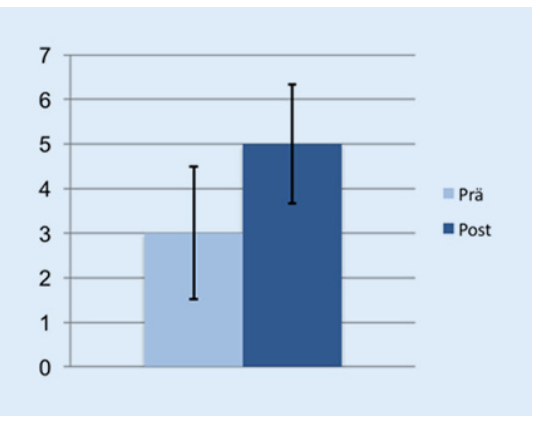

Abb. 3 ॥ Darstellung der Ergebnisse zur Frage "Wie schätzen Sie Ihre Fertigkeiten im Umgang mit individualisierter Medizin und Big Data ein?" im Prä-/Postvergleich (1 = sehr gering, $4=$ neutral, $7=$ sehr hoch)

gorie. Diese umfasst alle Aussagen über Lernerfolge der Studierenden im Umgang mit Daten im medizinischen Alltag und der Veränderung der Kommunikationsstrukturen. Die Unterkategorie Handlungsfähigkeit beschreibt in 11 Fällen einen Kompetenzzuwachs im Bereich der Handhabung digitaler Tools und die Auseinandersetzung mit den im Kurs erlebten Erfahrungen. In 6 Fällen erfolgt eine kritische und reflektierte Auseinandersetzung mit der veränderten $\mathrm{Ar}$ beitswelt. In $\square$ Abb. 1 sind die Ergebnisse der Evaluation in Form der semistrukturierten Interviews graphisch dargestellt, und Zitate der Studierenden werden den einzelnen Kategorien exemplarisch zugeordnet. Generell sagen die Teilnehmenden aus, nach Kursabschluss den Entwicklungen der digitalen Medizin sensibilisiert und differenziert gegenüberzustehen.

\section{Quantitative Prä-/Postevaluation Data Literacy}

Die quantitative Evaluation erfolgte getrennt für die Teilkompetenzen Wissen, Fertigkeiten und Haltung anhand einer siebenstufigen Likert-Skala in Bezug auf individualisierte Medizin und Big Data. Insgesamt lässt sich sagen, dass sich in der Prä-/Postevaluation eine positive Kompetenzentwicklung nachvollziehen lässt. Durch die Teilnahme konnte ein vorab geringer Wissenstand auf ein höheres Niveau gesteigert werden ( $\bullet$ Abb. 2). Geringe Vorfertigkeiten konnten im Rahmen der Unterrichtseinheit erweitert und verbessert werden (• Abb. 3). In Bezug auf

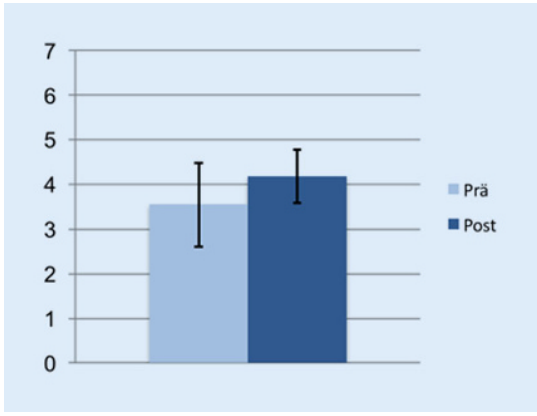

Abb. 4 A Darstellung der Ergebnisse zur Frage „Wie schätzen Sie Ihre Haltung/Einstellung zur individualisierten Medizin und Big Data ein?" im Prä-/Postvergleich ( 1 = sehr gering, $4=$ neutral, $7=$ sehr hoch)

Haltung stellt der Wert 3,5 eine neutrale Haltung dar, während niedrigere Werte eine ablehnende und höhere Werte eine positive Haltung ausdrücken. Die zu Beginn neutrale Haltung zeigte sich auch zum Abschluss des Unterrichts relativ konstant (• Abb. 4).

\section{Diskussion}

Die Digitalisierung der Medizin ist ein fundamentaler Wandlungsprozess, dessen Einfluss auf das zukünftige Berufsbild des Arztes noch nicht abgeschätzt werden kann. Jedoch steht fest, dass diese Entwicklung Lern- und Arbeitsprozesse wesentlich modifiziert und eine begleitende Ausbildung erfordert. Der Wandel von wissens- zu prozessbezogenem Denken muss auch im Studium reflektiert werden. Der notwendige Kompetenzerwerb verlangt eine grundlegende und aktive Auseinandersetzung mit den Kernthemen der digitalen Transformation [20].

Data Literacy wird aktuell als einer der führenden 21st Century Skills diskutiert [21]. Zum jetzigen Zeitpunkt findet dieser Kompetenzanspruch jedoch kaum Beachtung in der curricularen Realität. Auch der Nationale Kompetenzbasierte Lernzielkatalog Medizin und der Masterplan Medizinstudium 2020 adressieren diese Aspekte nicht $[22,23]$. Das Curriculum "Medizin im digitalen Zeitalter“ verfolgt den Ansatz, die digitale Transformation der Medizin transdisziplinär und interaktiv abzubilden. Neben der Teilkompetenz Wissen liegt hierbei ein Schwerpunkt auf den Aspekten Fertig- 
Hier steht eine Anzeige.

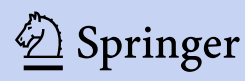


keiten und Haltung. Die Veränderungen in der Kompetenzbeurteilung - Wissen und Fertigkeiten - spiegeln dabei die bisherigen und neu erworbenen Erfahrungen der Kursteilnehmenden wider. Das eher geringe Vorwissen und die niedrigen Vorfertigkeiten zum Themenkomplex Data Literacy konnten in der Selbsteinschätzung der Studierenden innerhalb der Unterrichtsveranstaltung auf ein höheres Niveau gesteigert werden. Die vor der Unterrichtseinheit neutrale Haltung blieb auch zum Ende des Kurses relativ stabil.

Nachhaltiges Wissen lässt sich am Besten in situativen und sozialen Kontexten produzieren, da Wissensinhalte generell nicht als feste Einheiten im Gehirn abgespeichert werden können, sondern immer als Produkt des Zusammenspiels von Subjekt und Umwelt entstehen [24]. Für den gesamten Lernprozess ist es wichtig, dass Ziele zunächst gemeinsam erstellt und im Anschluss ebenso gemeinsam verfolgt und erreicht werden. Oftmals wird Data Literacy als Data Science missverstanden und den Bereichen Statistik/Mathematik/Informatik oder im medizinischen Kontext der medizinischen Informatik zugeordnet. Wie im vorliegenden Artikel skizziert, beschreibt der Begriff Data Literacy jedoch vielmehr den kompetenten Umgang mit Daten durch den Endanwender, d.h. die kritische und adäquate Interpretation und Anwendung der Daten durch den Arzt. Somit ist ein transdisziplinärer Ansatz erforderlich, der durch die Integration verschiedener Experten die Breite der digitalen Transformation der Medizin widerspiegelt, sich hierbei jedoch klar an der Sichtweise des Arztes orientiert. Medizin im digitalen Zeitalter ist das erste Curriculum in Deutschland, das diesen Ansatz abbildet.

\section{Fazit für die Praxis}

- Für die Medizineraus- und -weiterbildung stellt der Kompetenzerwerb der Data Literacy eine aktuelle Herausforderung dar.

- Das an der Universitätsmedizin Mainz durchgeführte Pilotprojekt hat gezeigt, dass die Integration diverser Fachexperten als Dozenten zielführend ist.

- Die Integration von Data Literacy in das Curriculum des Medizinstudiums und in die ärztliche Fort- und Weiterbildung ist eine transdisziplinäre und longitudinale Aufgabe.

- Bei der Entwicklung dieser Curricula sollte die hohe Geschwindigkeit des Veränderungsprozesses der digitalen Transformation beachtet und die curriculare Anpassung im Sinne eines Agility by Design bereits bei der Konzeption adressiert werden.

\section{Korrespondenzadresse}

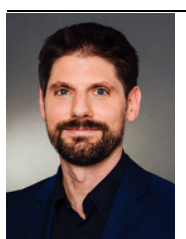

PD Dr. med. S. Kuhn, MME

Zentrum für Orthopädie und Unfallchirurgie, Universitätsmedizin der Johannes Gutenberg-

Universität

Langenbeckstr. 1, 55131 Mainz, Deutschland Sebastian.kuhn@unimedizinmainz.de

Förderung. Medizin im digitalen Zeitalter wird als curriculares Reformprojekt der Universitätsmedizin Mainz vom Stifterverband im Rahmen des mit der Carl-Zeiss-Stiftung gemeinsam initiierten Programms Curriculum 4.0 gefördert.

\section{Einhaltung ethischer Richtlinien}

Interessenkonflikt. S. Kuhn, D. Kadioglu, K. Deutsch und S. Michl geben an, dass kein Interessenkonflikt besteht.

Dieser Beitrag beinhaltet keine von den Autoren durchgeführten Studien an Menschen oder Tieren.

Open Access Dieser Artikel wird unter der Creative Commons Namensnennung 4.0 International Lizenz (http://creativecommons.org/licenses/by/4.0/deed. de) veröffentlicht, welche die Nutzung, Vervielfältigung, Bearbeitung, Verbreitung und Wiedergabe in jeglichem Medium und Format erlaubt, sofern Sie den/die ursprünglichen Autor(en) und die Quelle ordnungsgemäß nennen, einen Linkzur Creative Commons Lizenz beifügen und angeben, ob Änderungen vorgenommen wurden.

\section{Literatur}

1. Horak P et al (2017) Precision oncology based on omics data: the NCT Heidelberg experience. Int J Cancer 141:877-886

2. Bundesministerium für Bildung und Forschung (2017) Individualisierte Medizin. https://www. bmbf.de/de/individualisierte-medizin-378.html. Zugegriffen:5.Dez. 2017

3. Bundesministerium für Bildung und Forschung (2017) Digitalisierung in der Medizin. https://www.bmbf.de/de/digitalisierung-in-dermedizin-2897.html. Zugegriffen: 11. Dezember 2017

4. IBM (2017) IBM Watson for Onkology. https:// www.ibm.com/watson/health/oncology-andgenomics/oncology/. Zugegriffen: 05. Dezember 2017

5. Ada Digital Health Ltd (2017) Ada Health. https:// ada.com. Zugegriffen: 11. Dezember 2017

6. Kuhn S, Frankenhauser S, Tolks D (2017) Bundesgesundheitsblatt. https://doi.org/10.1007/s00103017-2673-z. Zugegriffen: 14. Dezember 2017

7. Persike M (2016) Lernen mit digitalen Medien aus Studierendenperspektive. Arbeitspapier Nr. 17. Hochschulforum Digitalisierung, Berlin

8. Kuhn S et al (2017) Medizin im digitalen Zeitalter: Was bedeutet das für die Ausbildung im Medizinstudium? Arbeitsmed Sozialmed Umweltmed 52(6):418-422

9. Hochschulforum Digitalisierung (2016) The Digital Turn - Hochschulbildung im digitalen Zeitalter. Arbeitspapier Nr. 27. Hochschulforum Digitalisierung, Berlin

10. Gilster P (1997) Digital literacy. John Wiley \& Sons, Inc, New York

11. Bawden D (2008) Origins and concepts of digital literacy. In: Lankshear C, Knobel M (Hrsg) Digital literacies: concept, policies, and practices. Peter Lang Publishing Inc, New York, S 17-32

12. Berkley University, Division of Data Science (2017) Engaging students in data science. https:// data.berkeley.edu/education. Zugegriffen: 15. Dezember 2017

13. NYU (2017) NYU University-wide initiative in data science. https://datascience.nyu.edu/about/. Zugegriffen: 15. Dezember 2017

14. Sapp Nelson M (2017) A pilot competency matrix for data management skills: a step toward the development of systematic data information literacy

15. Hochschulforum Digitalisierung (2017) Hochschulforum Digitalisierung Arbeitsgruppe Curriculum 4.0. https://hochschulforumdigitalisierung de/de/themen/curriculum-40. Zugegriffen: 14. Dezember 2017

16. Hochschulforum Digitalisierung (2017) Übergreifende Kompetenzen und Studieninhalte in der digitalen Welt am Beispiel von Data Literacy. https://hochschulforumdigitalisierung.de/de/ news/ausschreibung-data-literacy. Zugegriffen: 14. Dezember 2017

17. Kuhn S, Kirchgässner E, Deutsch K (2017) Medizin im digitalen Zeitalter. Do it by the book but be the author. Synergie 4:28-31. https://uhh.de/9ipxl. Zugegriffen: 14. Dezember 2017

18. Mayring P (2004) Qualitative Inhaltsanalyse. In: Flick U, von Kardoff E, Steinke I (Hrsg) Qualitative Forschung. Ein Handbuch. Rowohlt, Hamburg, S468-475

19. Mayring P (1997) Qualitative Inhaltsanalyse. Grundlagen und Techniken, 6. Aufl. Beltz, Weinheim

20. Kuhn S (2016) Medizin im digitalen Zeitalter - Ein Plädyer für Innovation und Kompetenzorientierung. https://hochschulforumdigitalisierung.de/ de/blog/medizin-digitalen-zeitalter-plaedoyerinnovation-kompetenzorientierung. Zugegriffen: 14. Dez. 2017

21. Ridsdale C, Rothwell J, Smit M (2015) trategies and best practices for data literacy education 
knowledge synthesis report. Dalhousie University, Halifax. http://www.mikesmit.com/wp-content/ papercite-data/pdf/data_literacy.pdf. Zugegriffen: 14. Dezember 2017

22. MFT Medizinischer Fakultätentag der Bundesrepublik Deutschland e.V. (2015) Nationaler Kompetenzbasierter Lernzielkatalog Medizin (NKLM). http://www.nklm.de/files/nklm_final_ 2015-07-03.pdf.Zugegriffen: 14. Dez. 2017

23. BMBF (2017) Masterplan Medizinstudium 2020. https://www.bmbf.de/files/2017-03-31_ Masterplan\%20Beschlusstext.pdf. Zugegriffen: 14. Dez. 2017

24. Tribelhorn T (2007) Situiertes Lernen in der Weiterbildung. In:WehrS, ErtelH(Hrsg)Aufbruch in der Hochschullehre. Kompetenzen und Lernende im Zentrum. Haupt, Bern, S31-76

\section{Neue Färbemethode ermöglicht Nano-CT-Aufnahmen von Gewebeproben}

Gewebeschnitte sind in Kliniken eine Standardprozedur, um beispielsweise Tumorgewebe zu untersuchen. Ein interdisziplinäres Team der Technischen Universität München (TUM) hat nun eine Färbemethode entwickelt, die es erlaubt, dreidimensionale Gewebeproben mit dem ebenfalls kürzlich an der TUM entwickelten Nano-CT-Gerät zu analysieren.

Bisher werden Gewebeproben von Patienten für histologische Untersuchungen in dünne Scheiben geschnitten, eingefärbt und unter dem Mikroskop analysiert. Ein großes Ziel der Medizin ist es, nicht nur Schnitte zu untersuchen sondern die gesamte dreidimensionale Probe. Eines der am besten geeigneten Verfahren dazu stellt die Computertomographie (CT) dar.

Bisher Einschränkungen bei Auflösung und Kontrast

Dass das Verfahren bisher nicht realisiert werden konnte, hat zwei Gründe: Erstens ist die Auflösung herkömmlicher CT-Geräte zu gering. Existierende Mikround Nano-CTs sind für den Einsatz in der Praxis selten geeignet. Bei manchen ist die Auflösung nicht variabel genug, andere sind auf Strahlung aus großen Teilchenbeschleunigern angewiesen. Zweitens lässt sich Weichgewebe schlecht in CT-Geräten untersuchen. Proben müssen eingefärbt werden, damit es überhaupt sichtbar wird. Färbemittel für CT-Aufnahmen sind teilweise sehr giftig und extrem zeitaufwendig in der Anwendung. Mitunter verändern sie das Gewebe so, dass es im Anschluss nicht weiter untersucht werden kann.

\section{Erfolgreiche Kooperation von} Physik, Chemie und Medizin

Wissenschaftler der Munich School of BioEngineering (MSB) an der TUM haben beide Probleme gelöst, indem sie ein NanoCT-Gerät entwickelten, das eine Auflösung von bis zu 100 Nanometern liefert und für den Betrieb in herkömmlichen Labors geeignet ist.

An einer Mausniere demonstrierten sie, dass im Nano-CT dreidimensionale Bilder erzeugt werden können, die dem Informationsgehalt von Gewebeschnitten entsprechen. Den Kern der Färbemethode bildet Eosin, der bislang als nicht CT-geeignet galt.
Um den Standard-Farbstoff für Gewebeschnitte nutzen zu können, ist unter anderem eine spezielle Vorbehandlung erforderlich. Das Färbeverfahren ist so zeitsparend, dass es auch im Klinikalltag anwendbar wäre. Ein großer Vorteil der Methode ist, dass die Gewebeprobe im Anschluss an den Scan problemlos mit klassischen Methoden weiter untersucht werden kann.

\section{Ergänzung statt Ersatz}

Als nächsten Schritt wollen die Forscher humane Gewebeproben untersuchen. In absehbarer Zeit wird CT-Histologie die klassischen Methoden jedoch nicht ersetzen. Das Team versteht das Verfahren zunächst als Ergänzung. Ärzte könnten so beispielsweise zusätzliche Informationen über die dreidimensionale Verteilung von Zellen und Zellkernen gewinnen.

Darüber hinaus werden neue Möglichkeiten für die medizinische Grundlagenforschung gesehen. Neben dem Einsatz in der Diagnose könnte die zerstörungsfreie und dreidimensionale Untersuchung mit dem NanoCT neue Einsichten in die mikroskopische Entstehung der Volkskrankheit Krebs liefern.

Literatur: M. Busse, M. Müller, M. A. Kimm et al (2018) PNAS. DOI: 10.1073/pnas.1720862115

Quelle: Technische Universität München, www.tum.de 